Gastrointestinal cancer

\title{
Observer variation in the diagnosis of superficial oesophageal adenocarcinoma: another spanner in the works?
}

\section{Alderson}

\section{The distinction between high grade dysplasia and intramucosal carcinoma is not easily made by expert pathologists, with little improvement even after agreeing on uniform criteria for the use of these terms}

\begin{abstract}
$\mathrm{T}$ he rapidly rising incidence of oesophageal adenocarcinoma in the Western world has stimulated considerable experimental and clinical research in recent years. After a long period of uncertainty, it is now generally held that the majority, if not all, of these cancers arise on a background of intestinal metaplasia (Barrett's oesophagus), secondary to chronic gastro-oesophageal reflux. While there has been much debate on the risk of cancer development, it is again accepted that in a proportion of patients, this metaplastic epithelium becomes unstable, leading to a sequential progression through dysplasia to carcinoma. Considerable effort has gone into characterising the changes which seem to occur at the molecular level ${ }^{1}$.
\end{abstract}

In this issue, Ormsby and colleagues suggest that it may be important to distinguish high grade dysplasia (HGD) from intramucosal carcinoma (IMC) in order to reach a decision about endoscopic surveillance and how this might influence the nature and timing of subsequent therapy [see page 671]. They show that the distinction between HGD and IMC is not easily made by expert pathologists, with little improvement even after agreeing on uniform criteria for the use of these terms. The biggest problem seems to be where the glandular architecture is complex, making the recognition of landmarks (basement membrane and muscularis mucosa) difficult. This seems to be a problem in at least $20 \%$ of cases where the pathologist is being specifically asked to separate HGD from IMC. The fact that they were working from resection specimens rather than small endoscopic biopsies probably means that this is an underestimate of the difficulties which most pathologists face.

Their paper raises a number of issues. Is it really important to separate HGD from IMC? Do the observations cast any doubt on the scientific validity of experimental studies which have tried to identify "biomarkers" of HGD? Is their own work open to criticism in terms of methodology and data interpretation?

It is easiest to deal with these questions in reverse order.

The study was carefully performed and the conclusion that experienced pathologists have difficulty distinguishing HGD from IMC is sound. Inevitably, this must mean that there are studies in which groups of "HGD patients" must include some patients with IMC. In fact, as far as experimental work is concerned, these always focus on markers of LGD (low grade dysplasia), HGD, or invasive cancer and none have sought to separate HGD from IMC ${ }^{1}$. As such, the current study probably does not invalidate our present state of knowledge regarding the molecular changes which seem to characterise the neoplastic process.

The real issue here is just how relevant is this distinction between HGD and IMC. The authors state that some groups "advocate oesophagectomy for a biopsy diagnosis of high grade dysplasia, others prefer to wait until intramucosal adenocarcinoma is detected". This is open to criticism. The two references chosen to support the latter point of view come from the same group of authors associated with the University of Washington in Seattle. Interestingly, the Seattle group have always sought to point out the difficulties in interpreting HGD with gross architectural distortion and have stated that in such cases "invasive adenocarcinoma cannot be excluded". As such, the assertion made by Ormsby et al that the distinction between HGD and IMC is important for day to day practice is less of an issue than they imply. Their work however undoubtedly indicates one of the reasons why we see such wide variation in the apparent behaviour of HGD and the risk of finding a carcinoma in an oesophagectomy specimen undertaken for HGD.

At the end of the day, management of patients with HGD largely reflects the way in which clinicians choose to interpret the available evidence and adhere to clinical protocols. Those who would wish to spare every patient an unnecessary oesophagectomy must have an underlying belief that a diagnosis of HGD is correct and the risk of invasive carcinoma is negligible. The pursuit of a surveillance policy for HGD must satisfy a number of criteria.

The endoscopic examination itself must be meticulous so that small raised lesions or ulcerated areas which are associated with an increased cancer risk are not overlooked. ${ }^{45}$ The biopsy protocol must be systematic, involving multiple biopsies of adequate size to minimise the risk of sampling error. The clinician must understand the difference in risk of a cancer being present when HGD is diagnosed at a prevalent rather than surveillance biopsy. Case series advocating long term non-surgical management of HGD on the basis of a low incidence of cancer development need to be considered carefully. Schnell and colleagues ${ }^{6}$ reported a five year cumulative cancer incidence of only $9 \%$, a figure well below that described by other groups using aggressive systematic biopsy protocols. ${ }^{4}$ Perhaps the clue to the low cancer incidence reported by Schnell et al was the high frequency with which dysplasia of any severity was identified. The inevitable inference is that many of the patients labelled as HGD would have been classified as LGD elsewhere. The problem of interobserver variation is once again raised. ${ }^{89}$

In the UK's healthcare system, clinicians frequently work in less than ideal circumstances. The inevitable consequence of an inadequate number of small biopsies will be the finding of an unsuspected cancer in specimens resected for HGD. A number of series published in the 1990s indicate that this risk is still approximately $40 \%{ }^{10}$ and unless clinicians can reassure themselves that they can deliver an aggressive surveillance protocol allied to consensus histopathology reporting with high levels of agreement, they should be recommending surgical resection for healthy patients with HGD.

Gut 2002;51:620-621

\section{Author's affiliation}

D Alderson, University Division of Surgery, Level 7, Bristol Royal Infirmary, Marlborough Street, Bristol BS2 8HW, UK ; derek.alderson@bristol.ac.uk

\section{REFERENCES}

1 Krishnadath KK, Reid BJ, Wang KK.

Biomarkers in Barrett esophagus. Mayo Clin Proc $2001 ; 76: 438-46$. 
2 Ormsby $\mathbf{A H}$ Petras RE, Henricks WH, et al. Observer variation in the diagnosis of superficial oesophageal adenocarcinoma. Gut 2002;51:671-6.

3 Levine DS, Haggitt RC, Blount PL, et al. An endoscopic biopsy protocol can differentiate high-grade dysplasia from early adenocarcinoma in Barrett's esophagus. Gastroenterology 1993;105:40-50.

4 Weston AP, Sharma P. Topalovski M, et al. Long-term follow-up of Barrett's high-grade dysplasia. Am J Gastroentero 2000;95: 1888-93

5 Montgomery E, Bronner MP, Greenson JK, et al. Are ulcers a marker for invasive carcinoma in Barrett's esophagus? Data from a diagnostic variability study with clinical follow-up. Am J Gastroentero 2002;97:27-31.

6 Schnell TG, Sontag SJ, Chejfec G, et al. Long-term nonsurgical management of Barrett's esophagus with high grade dysplasia. Gastroenterology 2001;120:1607-19.

7 Reid BJ, Levine DS, Longton G, et al. Predictors of progression to cancer in Barrett's esophagus: baseline histology and flow cytometry identify low- and high risk patient subsets. Am J Gastroenterol 2000;95:1669-76.
8 Skacel M, Petras RE, Gramlich TL, et al. The diagnosis of low-grade dysplasia in Barrett's esophagus and its implications for disease progression. Am J Gastroenterol 2000:95:3383-7.

9 Montgomery E, Bronner MP, Goldblum JR, et al. Reproducibility of the diagnosis of dysplasia in Barrett esophagus: a reaffirmation. Hum Pathol 2001;32:368-78.

10 Al-kasspooles MF, Hill HC, Nava HR, et al. High-grade dysplasia within Barrett's esophagus: Controversies regarding clinical opinions and approaches. Ann Surg Oncol 2002;9:222-7.
Endoscopic ultrasound is beyond dispute the best technique for locoregional staging - that is, assessment of the "vertical" extension of gastric lymphomasalthough it is still not employed in all patients. ${ }^{8}$ In our experience, upstaging was shown in $18 \%(4 / 22)$ of patients with low grade MALT lymphomas and in $36 \%(4 / 11)$ of patients with DLBCL with EUS based staging compared with computed tomography based staging. EUS however is not a sensitive technique to diagnose multifocality and horizontal extension of gastric MALT lymphoma.

Apart from the diagnostic value of EUS for locoregional staging, this technique also gives prognostic information on the probability that a histological remission may be obtained after $H$ pylori eradication. This response in gastric MALT-type lymphoma (low grade disease) is strongly related to the extent of involvement of the gastric wall, as shown by endoscopic ultrasound. ${ }^{5}$ If the lesions

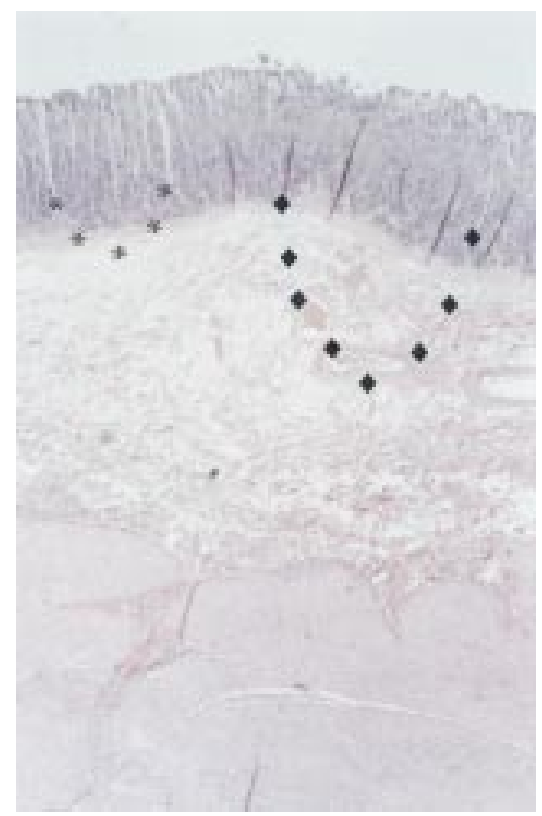

Figure 1 Depth of biopsy reached by a routine endoscopic biopsy forceps (left) and a large calibre forceps (right). The latter samples well into the muscularis mucosae and upper submucosa.
Clinical presentation and endoscopic findings in primary gastric lymphoma mic benign and other mati patients but exact subtyping of the lymphoma and the "horizontal" extension of the disease on a limited number of small endoscopic biopsies may be more difficult..$^{3-5}$ Therefore, a second gastroscopy for "intragastric staging" with an extensive biopsy protocol is strongly advocated using a treatment endoscope (for example, Olympus GIF-IT or 2T 100-160) and a large calibre biopsy forceps over the routine endoscopes, as used by others (fig 1). Gastric marginal zone lymphoma of the MALT-type is most often a multifocal disease. Significant foci of malignant large B cells (high grade disease) can be observed in 10-15\% of cases ${ }^{45}$ (de Jong and Boot unpublished). The need for a standardised protocol taking preferably 20-30 biopsies of both abnormal and seemingly uninvolved mucosa is therefore obvious in order to assess horizontal extension and multifocality, both initially and during follow up. Moreover, this will preclude overlooking minor components of transformed/high grade disease that have a negative prognostic impact, both in conventionally treated patients (radiotherapy, chemotherapy) and in patients treated with $H$ pylori eradication. $^{67}$ 
are confined to the mucosa and submucosa without lymph node involvement, in $\pm 80 \%$ of MALT-lymphomas (lowgrade) a histological remission may be obtained. ${ }^{5}$ After H pylori eradication, persistence of monoclonal bands has been described in otherwise unsuspicious basal lymphoid aggregates, despite the fact that histological remission has been obtained, ${ }^{9}$ indicating the deep mucosa and submucosa (second and third layer at conventional endoscopic ultrasonography) as regions of special interest. Non-responsiveness to $H$ pylori eradication has been attributed to the presence of DLBCL or high grade disease in the deeper layers of the gastric wall. ${ }^{10} \mathrm{Re}$ cently, the presence of the $t(11 ; 18)$ translocation was shown to be highly predictive of responsiveness to $\mathrm{H}$ pylori eradication. Histological complete remission was observed in $68 \%$ of 67 translocation negative cases, while in 44 translocation positive cases only two patients showed a transient histological remission. ${ }^{11}$

During follow up after $H$ pylori eradication in (low grade) MALT lymphoma, endoscopic abnormalities such as ulcers, erosions, and abnormal mucosal folds show marked improvement long before histological remission is documented. In these cases a prolonged follow up of 12-18 months may be necessary, at which time endoscopy is often (near) normal.

In most patients treated with radiotherapy, a radiation dose of 35-40 Gy in daily fractions of 1.8-2 Gy is applied. Assessment of the response with endoscopic biopsies should not be done too early as the response to radiotherapy may also occur slowly. We perform endoscopy two months after the end of radiotherapy. During prolonged follow up after radiation therapy, retraction of the lesser curve may occur due to fibrosis, especially if the primary lesion was located at the angulus or lesser curvature of the antrum.
Patients with DLBCL with or without MALT features can be treated with CHOP-like chemotherapy (cyclophosphamide, doxorubicin, vincristine, and prednisone). A response to chemotherapy in DLBCL may occur very rapidly.

The role of EUS during follow up is not yet determined and the timely study of Püspök et al addresses this topic. They described increased echogenicity during follow up EUS. We performed EUS during follow up of 26 patients and found a normal pattern in five, increased echogenicity in 14 (related to histologically confirmed fibrosis and scarring in 12 after radiotherapy), and increased hypoechoic thickening of the second and third layers in seven patients. In six of these patients recurrent or residual lymphoma after treatment was demonstrated histologically.

The implications of the study of Püspök et al are not easy to translate into clinical practice. In primary gastric lymphoma the response to $H$ pylori eradication may occur more slowly compared with chemotherapy. The value of EUS for follow up in gastric lymphoma seems limited and endoscopy with multiple biopsies remains the investigation of choice. If the first "staging" EUS shows thickened gastric wall layers, it seems prudent to use EUS during follow up after six months, especially when $H$ pylori eradication is used as the primary treatment. A component of DLBCL may be present in the deeper layers of the gastric wall, necessitating treatment adjustment. ${ }^{10}$ Moreover, EUS remains useful when endoscopic abnormalities are detected during follow up, especially thickened mucosal folds. Conventional histology remains the "gold standard" for documentation of a complete remission, as the significance of persistent monoclonality in patients with a histological remission remains unclear.'

Gut 2002;51:621-622

\section{Authors' affiliations}

H Boot, D de Jong, Department of Gastroenterology and Pathology, Antoni van Leeuwenhoek Hospital, Netherlands Cancer Institute, Plesmanlaan 121, $1066 \mathrm{CX}$ Amsterdam, the Netherlands

Correspondence to: Dr H Boot; h.boot@nki.nl

\section{REFERENCES}

1 Püspök A, Raderer $M$, Chott A, et al. Endoscopic ultrasound in the follow up and response assessment of patients with primary gastric lymphoma. Gut 2002;51:691-4.

2 Jaffe ES, Harris NL, Stein $\mathrm{H}$, et al. World Health Organisation classification of tumours: tumours of haematopoietic and lymphoid tissues. Lyon: IARC Press, 2001.

3 Taal BG, Boot $H$, van Heerde $P$, et al. Primary non-Hodgkin lymphoma of the stomach: endoscopic pattern and prognosis in low versus high grade malignancy in relation to the MALT concept. Gut 1996;39:556-61.

4 Fischbach W, Dragosics B, Kolve-Goebeler $M-E$, et al. Primary gastric B-cell lymphoma: Results of a prospective multicenter study. Gastroenterology 2000;1 19:1 191-202.

5 Ruskoné-Fourmestraux A, Lavergne A, Aegerter $\mathrm{PH}$, et al. Predictive factors for regression of gastric MALT lymphoma after anti-Helicobacter pylori treatment. Gut 2001;48:297-303

6 De Jong $D$, Boot $H$, van Heerde $P$, et al. Histological grading in gastric lymphoma: pretreatment criteria and clinical relevance. Gastroenterology 1997;1 12:1466-74.

7 De Jong D, Vyth-Dreese F, Dellemijn T, et al. Histological and immunological parameters to predict treatment outcome of Helicobacter pylori eradication in low-grade gastric MALT lymphoma. J Pathol 2001;193:318-24.

8 De Jong D, Aleman BMP, Taal BG, et al. Controversies and consensus in the diagnosis, work-up and treatment of gastric lymphoma: An international survey. Ann Oncol 1999; 10:275-80.

9 Thiede Chr, Wündisch Th, Alpen B, et al. Longterm persistence of monoclonal $B$ cells after cure of Helicobacter pylori infection and complete histological remission in gastric mucosa-associated lymphoid tissue B-cell lymphoma. J Clin Oncol 2001;19:1600-9.

10 Bayerdörffer E, Neubauer A, Rudolph B, et al. Regression of primary gastric lymphoma of mucosa-associated lymphoid tissue type after cure of Helicobacter pylori infection. Lancet 1995; 1345: 1591-4.

11 Liu H, Ye A, Ruskoné-Fourmesrtaux A, et al $T(1) ; 18)$ is a marker for all stage gastric MALT lymphomas that not will respond to $H$. pylori eradication. Gastroenterology 2002; 1 22: 1286-94. 